\title{
On a possible explanation of the DLS puzzle
}

\author{
M. Bashkanov ${ }^{1,2}$ and H. Clement ${ }^{1,2, a}$ \\ 1 Physikalisches Institut, Eberhard-Karls-Universität Tübingen, Auf der Morgenstelle 14, D-72076 Tübingen, Germany \\ 2 Kepler Center for Astro and Particle Physics, University of Tübingen, Germany
}

Received: 9 April 2014

Published online: 4 July 2014

(C) The Author(s) 2014. This article is published with open access at Springerlink.com

Communicated by H. Ströher

\begin{abstract}
The enhancement in the dilepton spectrum observed in heavy-ion collisions for invariant electronpositron masses in the range $0.15 \mathrm{GeV}<M_{e^{+} e^{-}}<0.6 \mathrm{GeV}$ has recently been traced back to a corresponding enhancement in $n p$ collisions relative to $p p$ collisions. Whereas the dilepton spectra in the latter are understood quantitatively, theoretical descriptions fail to describe the much higher dilepton rate in $n p$ collisions at beam energies below $2 \mathrm{GeV}$, in particular regarding the region $M_{e^{+} e^{-}}>0.3 \mathrm{GeV}$. We show that the missing strength can be attributed to $\rho^{0}$-channel $\pi^{+} \pi^{-}$production, which is dominated by the $t$-channel $\Delta \Delta$ excitation and the recently found isoscalar dibaryonic resonance structure at $2.37 \mathrm{GeV}$. These processes do not contribute in $p p$ collisions, but only in $n p$ collisions.
\end{abstract}

\section{Introduction}

Dilepton spectroscopy has been established as a valuable tool to explore the conditions of matter at high temperature and high density. Such extreme conditions as found in stars or in the early universe can be probed by relativistic heavy-ion collisions. In measurements of such collision processes a significant excess of lepton pairs over the theoretically expected rate has been observed in the mass region between the pion mass and the $\omega$ mass and interpreted as a possible sign of medium modifications. However, at lower beam energies of (1-2) GeV per nucleon still such an enhancement has been observed.

To address this problem the Dilepton Spectrometer (DLS) Collaboration was the first to investigate the underlying basic reactions by studying the dilepton production in $p p$ and $p n$ collisions - the latter by use of $d p$ collisions. As a result they found that an enhancement persists even for beam energies as low as $1 \mathrm{GeV}$ ("DLS puzzle") [1,2]. In recent measurements of dilepton pairs produced in $\mathrm{C}+\mathrm{C}$, $p+n$ and $p+p$ collisions at HADES the enhancement observed in heavy-ion collisions could be traced back to such a one in $p n$ relative to $p p$ collisions processes [3]. A number of theoretical calculations have been successful in explaining the dilepton spectrum originating from $p p$ collisions. They also succeeded in predicting a significantly higher dilepton rate for $p n$ collisions. However, they all underpredict the $p n$-induced dilepton production for $M_{e^{+} e^{-}}>0.3 \mathrm{GeV}$ by up to an order of magnitude [4-7] at

\footnotetext{
a e-mail: clement@pit.physik.uni-tuebingen.de
}

beam energies below $2 \mathrm{GeV}$, though ref. [4] can cure much of the disagreement by introduction of a pion electromagnetic form factor.

In these calculations the following lepton-pair production processes have been taken into account:

- pion Dalitz decay $\pi^{0} \rightarrow e^{+} e^{-} \gamma$;

- $\eta$ Dalitz decay $\eta \rightarrow e^{+} e^{-} \gamma$

- leptonic vector meson decay $\rho / \omega \rightarrow e^{+} e^{-}$;

- virtual bremsstrahlung $N N \rightarrow N N e^{+} e^{-}$and

- baryon resonance decay $R \rightarrow N e^{+} e^{-}$, predominantly $\Delta \rightarrow N e^{+} e^{-}$.

The bremsstrahlung calculations of ref. [8] overshoot the HADES data for $p p$ collisions. For the $n p$ case they overshoot the data for $M_{e^{+} e^{-}}<0.3 \mathrm{GeV}$ and underpredict them above.

At sufficiently high incident energies both colliding nucleons may get excited. So in addition to the configuration $N R$, where $R$ denotes a nucleon in one of its excited states (resonance), we may have combinations of the form $R R^{\prime}$. The lowest-lying such configuration is $\Delta \Delta$. In the following we will concentrate on the beam energy $1.25 \mathrm{GeV}$, where high-precision HADES data are available. As we will argue below the only relevant $R R^{\prime}$ configuration there is $\Delta \Delta$.

At the energies of interest here, single-pion production in $N N$ collisions is by far the largest inelastic channel. It is dominated by $t$-channel meson exchange in combination with the excitation of one of the nucleons into the $\Delta(1232)$ resonance - or to a lesser extent to the Roper resonance $N^{*}(1440)$ with subsequent decay into the $\pi N$ system. 
In the description of the dilepton spectra two-pion production has not been taken into account in most of the previous works, since its cross section is smaller by an order of magnitude. However, as we will show in the following, due to the relatively large decay branch $\rho^{0} \rightarrow e^{+} e^{-}$the $\rho$ production in the $\Delta \Delta$-channel contributes significantly to the electron-positron spectrum for $M_{e^{+} e^{-}} \geq 0.3 \mathrm{GeV}$.

In ref. [5] two-pion production has been accounted for in a global manner. Here we proceed differently. Since the two-pion channels have been investigated experimentally meanwhile by exclusive and kinematically complete measurements, we know the dominating two-pion production mechanisms in dependence of the energy in detail. In particular, we are able to perform an isospin decomposition of experimental cross sections and underlying reaction mechanisms, in order to separate their contributions to $p p$ - and $p n$-induced dilepton production.

\section{Two-pion production}

In recent years the two-pion production in $p p$ and $p n$ collisions has been measured by exclusive and kinematically complete experiments over the energy region from threshold up to $T_{\text {lab }}=1.4 \mathrm{GeV}[9-23]$.

It has been shown that the $p p$-induced two-pion production process is dominated by $t$-channel Roper and $\Delta \Delta$ excitation [9-19,24,25]. In the latter both nucleons are mutually excited to the $\Delta$ resonance by $t$-channel meson exchange in the collision process. The Roper excitation process dominates at beam energies close to threshold below $1 \mathrm{GeV}$, whereas the $\Delta \Delta$ process takes over above $1 \mathrm{GeV}$. Hence in the following we will focus on the latter two-pion production process. And since the HADES experiment has been carried out at $T_{p}=1.25 \mathrm{GeV}$, we will concentrate on this energy.

Also, in $p n$-induced two-pion production the recently discovered dibaryon resonance structure $d^{*}$ with $I\left(J^{P}\right)=$ $0\left(3^{+}\right), M=2.37 \mathrm{GeV}$ and $\Gamma=70 \mathrm{MeV}$ strongly contributes at energies around $1.2 \mathrm{GeV}$ due to its decay $d^{*} \rightarrow \Delta \Delta \rightarrow N N \pi \pi[20-23,26]$.

The total inclusive cross section for $p p$-induced $\pi^{+} \pi^{-}$ production at $T_{p}=1.25 \mathrm{GeV}$ is about $700 \mu \mathrm{b}$ and for $n p$ induced $\pi^{+} \pi^{-}$production it is about $1300 \mu \mathrm{b}$. The latter contains not only the $n p \pi^{+} \pi^{-}$-channel, but also the double-pionic fusion channel $d \pi^{+} \pi^{-}$.

The only sizeable way two-pion production may feed the electron-pair production is via $\pi^{+} \pi^{-} \rightarrow \rho^{0} \rightarrow e^{+} e^{-}$ with the isovector $\pi^{+} \pi^{-}$pair being in relative $p$-wave $(\rho$ channel).

In order to filter out the $\rho$-channel $\pi^{+} \pi^{-}$production from the known two-pion production cross sections, we make use of the isospin decomposition of these cross sections in terms of matrix elements $M_{I_{N N}^{f} I_{\pi \pi} I_{N N}^{i}}$, where $I_{\pi \pi}$ stands for the isospin of the pion pair and $I_{N N}^{i}$ and $I_{N N}^{f}$ for the isospin of the nucleon pair in initial and final states, respectively $[27,28,13]$.

For a specific process these matrix elements depend on the isospin coupling coefficients. For the $\Delta \Delta$ process the matrix elements are proportional to the respective $9 j$ symbol for isospin recoupling:

$$
M_{I_{N N}^{f} I_{\pi \pi} I_{N N}^{i}}^{\Delta \Delta} \sim \hat{I_{\Delta_{1}} I_{\Delta_{2}} I_{N N} \hat{I_{\pi \pi}}}\left\{\begin{array}{ccc}
I_{N_{1}} & I_{\pi_{1}} & I_{\Delta_{1}} \\
I_{N_{2}} & I_{\pi_{2}} & I_{\Delta_{2}} \\
I_{N N} & I_{\pi \pi} & I_{\Delta \Delta}
\end{array}\right\}
$$

where $N_{i}$ and $\pi_{i}$ couple to $\Delta_{i}$ for $i=1,2$ and $\hat{I_{\alpha}}=$ $\sqrt{2 I_{\alpha}+1}$.

In $p p$-initiated two-pion production only $M_{111}$ gives rise to $\rho^{0}$-channel production. However, $M_{11}^{\Delta \Delta} \equiv 0$ for the $\Delta \Delta$ process, since the corresponding $9 j$-symbol in eq. (1) vanishes. Hence there is no contribution from $\Delta \Delta$ excitation to $\rho^{0}$-channel production. Therefore, the Pluto [29] generated cocktail for the description of the $p p$ dilepton production as given in ref. [3] stays unchanged.

The situation changes dramatically in case of $p n$ initiated $\rho^{0}$-channel production, since here we indeed do have large contributions from the $\Delta \Delta$ process. According to refs. $[27,28]$ we have for the $p n$-initiated $\pi^{+} \pi^{-}$production:

$$
\begin{aligned}
\sigma\left(p n \rightarrow p n \pi^{+} \pi^{-}\right)= & \frac{1}{60}\left|\sqrt{5} M_{101}-M_{121}\right|^{2} \\
& +\frac{1}{8}\left|M_{011}\right|^{2}+\frac{1}{24}\left|M_{110}\right|^{2} \\
& +\frac{1}{12}\left|M_{000}\right|^{2}
\end{aligned}
$$

and since $I_{d}=0$

$$
\sigma\left(p n \rightarrow d \pi^{+} \pi^{-}\right)=\frac{1}{8}\left|M_{011}\right|^{2}+\frac{1}{12}\left|M_{000}\right|^{2} .
$$

For dilepton production via $\rho^{0}$ production only matrix elements with $I_{\pi \pi}=1$ contribute. Selecting in addition the $\Delta \Delta$ process we end up with

$$
\sigma\left(p n \rightarrow \Delta \Delta \rightarrow p n\left[\pi^{+} \pi^{-}\right]_{I=1}\right)=\frac{1}{8}\left|M_{011}^{\Delta \Delta}\right|^{2}+\frac{1}{24}\left|M_{110}^{\Delta \Delta}\right|^{2}
$$

and

$$
\sigma\left(p n \rightarrow \Delta \Delta \rightarrow d\left[\pi^{+} \pi^{-}\right]_{I=1}\right)=\frac{1}{8}\left|M_{011}^{\Delta \Delta}\right|^{2} .
$$

With the relations

$$
M_{011}^{\Delta \Delta}=\sqrt{\frac{5}{12}} M_{110}^{\Delta \Delta}=\sqrt{\frac{15}{2}} M_{121}^{\Delta \Delta},
$$

obtained by angular momentum recoupling according to eq. (1), this leads to

$$
\begin{aligned}
& \sigma\left(p n \rightarrow \Delta \Delta \rightarrow p n\left[\pi^{+} \pi^{-}\right]_{I=1}\right)=\frac{27}{16}\left|M_{121}^{\Delta \Delta}\right|^{2} \\
& =\frac{45}{4} \sigma\left(p p \rightarrow \Delta \Delta \rightarrow n n \pi^{+} \pi^{+}\right)
\end{aligned}
$$

since $[27,28]$

$$
\sigma\left(p p \rightarrow n n \pi^{+} \pi^{+}\right)=\frac{3}{20}\left|M_{121}\right|^{2} .
$$


The analysis of the $p p \rightarrow n n \pi^{+} \pi^{+}$reaction gives about $15 \mu \mathrm{b}$ [16] for this cross section at $T_{p}=1.25 \mathrm{GeV}$, which results in

$$
\sigma\left(p n \rightarrow \Delta \Delta \rightarrow p n\left[\pi^{+} \pi^{-}\right]_{I=1}\right) \approx 170 \mu \mathrm{b} .
$$

This value roughly corresponds to one fourth of the full $\Delta \Delta$ production in the $p n \rightarrow p n \pi^{+} \pi^{-}$reaction [24].

In case the final $p n$ pair fuses to a deuteron we also obtain $\rho^{0}$-channel production, which is related to the measured $\pi^{+} \pi^{0}\left(\rho^{+}\right.$-channel $)$production in $p p$ collisions by the isospin relation [22]

$$
\sigma\left(p n \rightarrow d\left[\pi^{+} \pi^{-}\right]_{I=1}\right)=\frac{1}{2} \sigma\left(p p \rightarrow d \pi^{+} \pi^{0}\right) \approx 100 \mu \mathrm{b} .
$$

In addition, the $\rho^{0}$-channel production in $p n$-initiated two-pion production is fed by excitation and decay of the $d^{*}$-resonance. Since its decay proceeds again via the $\Delta \Delta$ system in the intermediate step, we can use the isospin relation for the $\Delta \Delta$ system —utilizing again eq. (1) -

$$
M_{110}^{\Delta \Delta}=-\sqrt{\frac{1}{2}} M_{000}^{\Delta \Delta},
$$

in order to connect the $p n\left[\pi^{+} \pi^{-}\right]_{I=1}$ decay channel with the $p n \pi^{0} \pi^{0}$-channel. Isospin-decomposed we have $[27,28]$

$$
\sigma\left(p n \rightarrow p n \pi^{0} \pi^{0}\right)=\frac{1}{30}\left|\frac{\sqrt{5}}{2} M_{101}+M_{121}\right|^{2}+\frac{1}{24}\left|M_{000}\right|^{2}
$$

and, for $I_{N N}^{i}=I_{d^{*}}=0$,

$$
\sigma\left(p n \rightarrow d^{*} \rightarrow d \pi^{0} \pi^{0}\right)=\frac{1}{24}\left|M_{000}\right|^{2} .
$$

At the resonance maximum at $\sqrt{s}=2.37 \mathrm{GeV}$ this cross section is about $240 \mu \mathrm{b}$, however, at $\sqrt{s}=2.42 \mathrm{GeV}$ $\left(T_{p}=1.25 \mathrm{GeV}\right)$ it is already as low as $90 \mu \mathrm{b}$. According to the predictions in refs. $[30,31]$ the resonance effect in the $p n \pi^{0} \pi^{0}$-channel should be $85 \%$ of that in the $d \pi^{0} \pi^{0}$ channel, i.e. about $80 \mu \mathrm{b}$. Together with eqs. (2), (11) and (13) and the condition $I_{N N}^{i}=I_{d^{*}}=0$ this results in

$$
\begin{aligned}
\sigma\left(p n \rightarrow d^{*} \rightarrow p n\left[\pi^{+} \pi^{-}\right]_{I=1}\right) & =\frac{1}{24}\left|M_{110}^{\Delta \Delta}\left(d^{*}\right)\right|^{2} \\
& =\frac{1}{48}\left|M_{000}^{\Delta \Delta}\left(d^{*}\right)\right|^{2} \\
& =\frac{1}{2} \sigma\left(p n \rightarrow d^{*} \rightarrow p n \pi^{0} \pi^{0}\right) \\
& \approx 40 \mu \mathrm{b} .
\end{aligned}
$$

A cross-check of this number is provided by a recent measurement [23] of the $p p \pi^{0} \pi^{-}$-channel, since again by isospin relations we have $[23,27,28]$,

$\sigma\left(p n \rightarrow d^{*} \rightarrow p n\left[\pi^{+} \pi^{-}\right]_{I=1}\right)==\sigma\left(p n \rightarrow d^{*} \rightarrow p p \pi^{0} \pi^{-}\right)$.
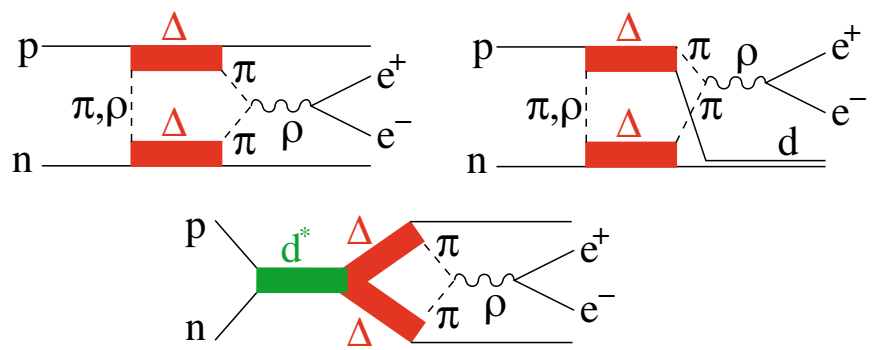

Fig. 1. (Color online) Graphs for the $e^{+} e^{-}$production via $\rho^{0}$-channel $\pi^{+} \pi^{-}$production in pn collisions. Top: Production via $t$-channel $\Delta \Delta$ excitation leading to $p n$ (left) and deuteron (right) final states. Bottom: Production via $s$-channel $d^{*}$ formation and its subsequent decay into the $\Delta \Delta$ system.

Though according to Gal and Garcilazo [32,33] the $d^{*}$ decay into isovector nucleon and pion pairs should be dynamically suppressed, the measurement of the $p n \rightarrow$ $d^{*} \rightarrow p p \pi^{0} \pi^{-}$reaction and its analysis [23] is compatible with a resonance cross section as expected by the isospin relations.

In total we have about $310 \mu \mathrm{b}$ of $\rho^{0}$-channel $\pi^{+} \pi^{-}$production in $p n$-initiated reactions at $T_{p}=1.25 \mathrm{GeV}$ compared to none in $p p$-initiated reactions. We estimate this number to be correct within $20 \%$.

\section{$3 \rho^{0}$-channel $\mathrm{e}^{+} \mathrm{e}^{-}$production}

To calculate the $e^{+} e^{-}$production we assume that the two pions produced in the $\Delta \Delta$ process undergo final-state interaction by forming a $\rho^{0}$, which subsequently decays into a $e^{+} e^{-}$pair:

$$
p n \rightarrow \Delta \Delta \rightarrow p n\left[\pi^{+} \pi^{-}\right]_{I=L=1} \rightarrow p n \rho^{0} \rightarrow p n e^{+} e^{-},
$$

see graphs in fig. 1 . The intermediate $\Delta \Delta$ system is formed either by $t$-channel meson exchange or by decay of the $d^{*}$ resonance with cross sections as evaluated above.

In both processes the $\Delta \mathrm{s}$ are formed side by side and due to their short lifetime the pions emitted in the $\Delta$ decay will be within their hadronic interaction range and undergo final-state interaction. From the $\pi \pi$ phaseshifts we know that the $I=L=1$ channel is very well described by just the $\rho$ resonance, which means that practically all $\pi^{+} \pi^{-}$pairs produced in this channel in the relevant energy regime will undergo $\rho$ formation.

For the transition from the $\left[\pi^{+} \pi^{-}\right]_{I=L=1}$ system into the $\left[e^{+} e^{-}\right]_{L=0}$ system by rescattering (final-state interaction in the $\rho$-channel) we use therefore a Breit-Wigner ansatz as used already in refs. [34,35]:

$$
\left|\mathcal{M}\left(\pi^{+} \pi^{-} \rightarrow \rho^{0} \rightarrow e^{+} e^{-}\right)\right|^{2}=\frac{m_{\rho}^{2} \Gamma_{\pi^{+} \pi^{-}} \Gamma_{e^{+} e^{-}}}{\left(s-m_{\rho}^{2}\right)^{2}+m_{\rho}^{2} \Gamma_{\rho}^{2}} .
$$

For the $p$-wave decay into the $\pi^{+} \pi^{-}$-channel we have $\Gamma_{\pi^{+} \pi^{-}} \sim q^{3}$ and for the $s$-wave decay into the $e^{+} e^{-}$channel we have $\Gamma_{e^{+}} e^{-} \sim k$, where $q$ and $k$ are the momenta in $\pi^{+} \pi^{-}$and $e^{+} e^{-}$subsystems, respectively. In a 
more detailed consideration [35] the partial widths depend also on the invariant masses $M_{\pi^{+} \pi^{-}}$and $M_{e^{+} e^{-}}$yielding $\Gamma_{\pi^{+} \pi^{-}}=a q^{3} / M_{\pi^{+} \pi^{-}}$and $\Gamma_{e^{+} e^{-}}=b k / M_{e^{+} e^{-}}^{3}$. The constants $a$ and $b$ in the partial widths are fixed by adjusting them to the known branching ratios and widths at the $\rho$ mass pole [36]. Hence the calculation of process (16) is straightforward and free of parameters.

The calculations have been carried out by Monte Carlo (MC) technique as it has been utilized before in refs. [924] for the description of the two-pion channels. Since the models used for calculating the processes depicted in fig. 1 have been tuned to reproduce integral and differential cross sections of the two-pion production measurements [13-17,20-23], this means that in essence we map the experimental results for $\rho$-channel two-pion production onto the $e^{+} e^{-}$-channel by means of eq. (17).

\section{Results}

The numerical results of these MC simulations are displayed in fig. 2. Since the HADES $n p$ data have not been taken with a pure neutron beam, but in quasi-free kinematics with the impinging neutron residing in the beam deuteron, the obtained invariant-mass spectra for the $n p$ case are Fermi smeared, which is taken into account in the simulations. Note that the neutrons' Fermi motion also leads to a larger high-mass limit of the measured dilepton spectrum in the $n p$ case compared to the $p p$ case.

At the top panel of fig. 2 we first show the $\rho^{0}$-channel $\pi^{+} \pi^{-}$spectrum obtained from the processes discussed in eqs. (1)-(15), solely scaled by the $e^{+} e^{-}$branching ratio at the pole of $\rho^{0}$ (dotted line), i.e. without using eq. (17). This gives just a very crude qualitative estimate. A proper quantum mechanical treatment has, of course, to involve the momentum-dependent transition amplitude in eq. (17). This then leads to the solid curve. The enhanced yield of the $e^{+} e^{-}$spectrum relative to the scaled $\pi^{+} \pi^{-}$ spectrum at low masses is due to the fact that - in addition to the inverse power dependence on the invariant mass - the pion pair is in relative $p$-wave and therefore suppressed near threshold, whereas the $e^{+} e^{-}$pair is in relative $s$-wave and hence not suppressed. The resulting integral cross section for the process $p n \rightarrow e^{+} e^{-} X$ is $72 \mathrm{nb}$, which is about a factor of four larger than that from the crude estimate.

Since we base here the dilepton production due to $\rho^{0}$ channel $\pi^{+} \pi^{-}$production on experimental results for the relevant two-pion production channels, we consider here only the on-shell situation. However, because the twolepton threshold is much lower than the two-pion threshold, also dilepton production via virtual $\rho^{0}$ formation in the intermediate state will contribute. Taking this into account removes the cut in the $e^{+} e^{-}$spectrum at the $\pi^{+} \pi^{-}$ threshold and replaces it by a smooth continuation as depicted, e.g., in fig. 3 of ref. [5]. Hence accounting for this will fill up the gap below $0.3 \mathrm{GeV}$ - possibly overshoot it even somewhat. We refrain here from doing such a calculation, since in contrast to the on-shell consideration pursued here the off-shell contribution is model-dependent.
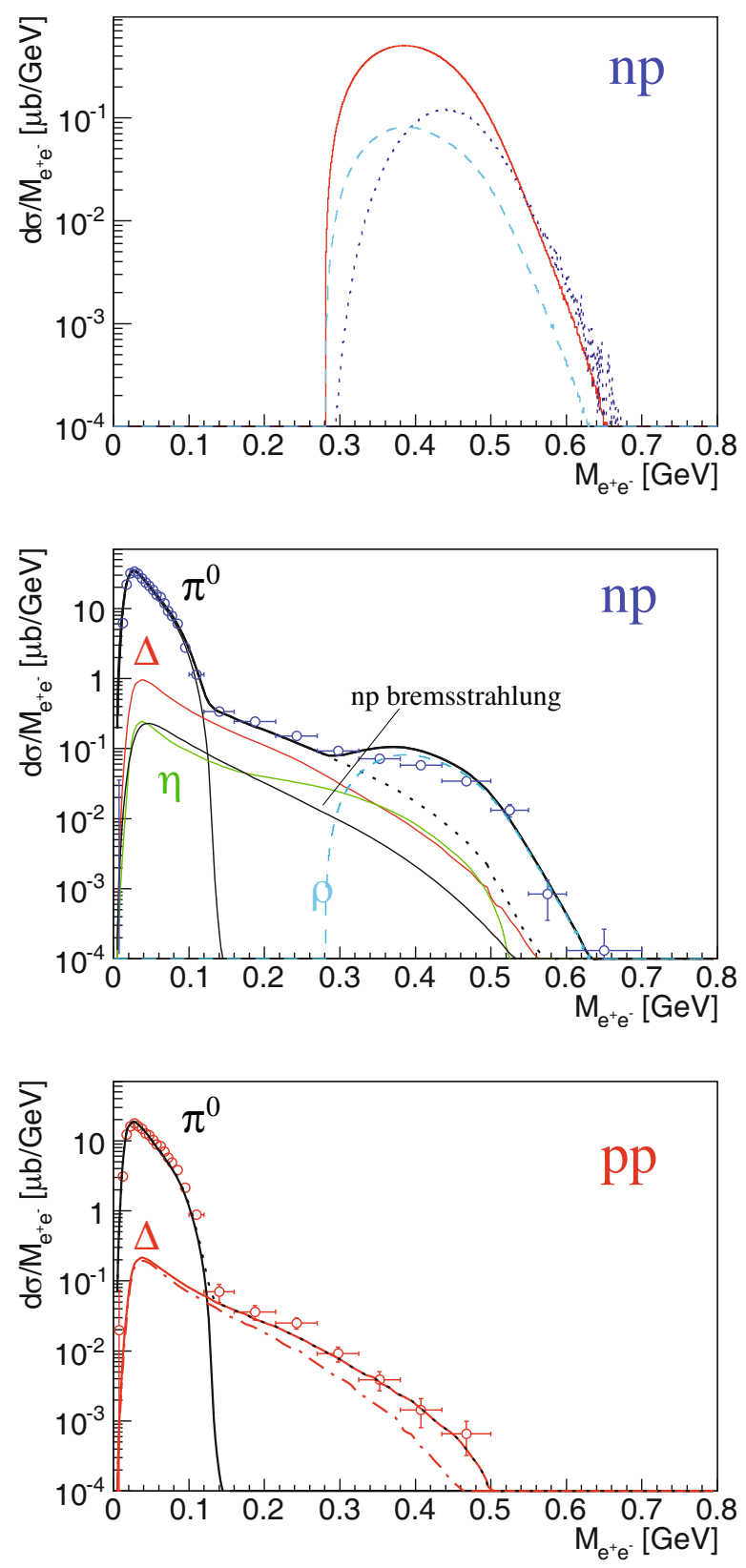

Fig. 2. (Color online) Distribution of the invariant mass $M_{e^{+} e^{-}}$produced in $N N$ collisions at $T_{p}=1.25 \mathrm{GeV}$. Top: $e^{+} e^{-}$production from $\rho^{0}$ decay resulting from the $\Delta \Delta$ excitation via on-shell $\pi^{+} \pi^{-}$production according to process (16). The drawn curves denote the $\left[\pi^{+} \pi^{-}\right]_{I=J=1}$ spectrum scaled by the $e^{+} e^{-}$branching ratio at the pole of $\rho^{0}$ (blue dotted), the resulting $e^{+} e^{-}$spectrum using the proper momentumdependent branching ratio (red solid) and the resulting $e^{+} e^{-}$ spectrum within the HADES acceptance (cyan dashed). Middle: Full $e^{+} e^{-}$production in $p n$ collisions. The open circles give the HADES result [3]. Thin solid lines denote calculations for $e^{+} e^{-}$production originating from $\pi^{0}$ production and bremsstrahlung (black), single $\Delta$ (VMD form factor, red) and $\eta$ (green) production with subsequent Dalitz decay. The dotted curve denotes the sum of these processes. The dashed (cyan) curve gives the contribution from the $\rho^{0}$-channel $\pi^{+} \pi^{-}$production and the thick solid line the sum of all these processes. Bottom: The same as above, but for $p p$ collisions. 
Since the HADES detector has limited acceptance, this has to be taken into account for comparison with the HADES data. The dashed curve exhibits the final $e^{+} e^{-}$ production resulting from $\rho^{0}$-channel $\pi^{+} \pi^{-}$production in $n p$ collisions within the HADES acceptance.

All other conventional processes due to $\pi^{0}, \eta$ and $\Delta$ Dalitz decays and bremsstrahlung - mentioned in the introduction - were simulated using the Pluto generator [29] and filtered with HADES efficiency-acceptance filters. Note that in Pluto the bremsstrahlung contributions are taken according to the prescription of ref. [4], which leads to a good description of the HADES data for the $p p$ case in contrast to that of ref. [8].

The calculations are shown in fig. 2(middle), in comparison with the HADES data for $n p$-initiated $e^{+} e^{-}$production at $T_{p}=1.25 \mathrm{GeV}$. The incoherent sum of these processes resulting from Dalitz decays is denoted by the dotted curve. It provides a quantitative description of the data in the region of the $\pi^{0}$ peak, i.e. for $M_{e^{+} e^{-}}<$ $0.15 \mathrm{GeV}$. Above, the sum curve under-predicts the data increasingly with increasing $M_{e^{+} e^{-}}$values. However, if we add the $e^{+} e^{-}$production resulting from $\rho^{0}$-channel $\pi^{+} \pi^{-}$ production (dashed curve both in top and middle parts of fig. 2) we obtain a nearly perfect description of the HADES data.

There appears still a slightly underestimated region in the range $0.15 \mathrm{GeV}<M_{e^{+} e^{-}}<0.3 \mathrm{GeV}$. It possibly might be related to direct $d^{*}$ decay $p n \rightarrow d^{*} \rightarrow d e^{+} e^{-}$ or $p n \rightarrow d^{*} \rightarrow[p n]_{I=0} e^{+} e^{-}$as suggested in ref. [6]. However, since we know neither shape nor strength of such a $d^{*}$ form factor in this channel, we cannot estimate such a contribution reliably. In addition, also the Pluto generated processes have theoretical uncertainties, which are in the order of the deviation in question. E.g., when treating $\Delta$ Dalitz and bremsstrahlung coherently, the high-mass range is affected noticeably [8].

For completeness we show at the bottom of fig. 2 also our results for the $p p$-initiated $e^{+} e^{-}$spectrum in comparison with the corresponding HADES data. Since in this case - as pointed out in the sect. 2 - there is no contribution from the $\Delta \Delta$ two-pion production, our results agree with those shown in ref. [3].

Finally we shortly comment on the dependence of the $e^{+} e^{-}$spectrum on the beam energy. The DLS Collaboration has measured the $e^{+} e^{-}$production in $p p$ and $p d$ collisions at several beam energies between 1.04 and $4.88 \mathrm{GeV}$. The ratio $R=\frac{Y_{p d}}{Y_{p p}}$ of integrated yields for $M_{e^{+} e^{-}}>$ $0.15 \mathrm{GeV}$ exhibits a peak-like structure with a substantial rise from $R \approx 6$ to $R \approx 9$ between $T_{p}=(1.0-1.27) \mathrm{GeV}$, falling off thereafter by a factor of roughly three until $T_{p}=2 \mathrm{GeV}$. At $2.1 \mathrm{GeV}$ the ratio is somewhat above 2 and at $4.9 \mathrm{GeV}$ a little bit below 2 .

Assuming the $p d$ collisions to proceed mainly as quasifree proton-nucleon collisions, we expect a ratio of $R=$ 2 , if $p p$ and $p n$ collisions contribute equal amounts. In the quasi-free picture the peak region corresponds to $2.3 \mathrm{GeV}<\sqrt{s}<2.7 \mathrm{GeV}$ with the maximum around $\sqrt{s} \approx 2.4 \mathrm{GeV}$, i.e. just in the region, where both the $d^{*}$ resonance formation and the $p n \rightarrow \Delta \Delta \rightarrow d\left[\pi^{+} \pi^{-}\right]_{I=1}$ process peak. Whereas the first one with a width of
$70 \mathrm{MeV}$ fades away below $\sqrt{s}=2.3 \mathrm{GeV}$ and above $\sqrt{s}=$ $2.5 \mathrm{GeV}$, the latter one with a width of about $250 \mathrm{MeV}[14$, 22 ] declines much slower fading away above $\sqrt{s}=2.8 \mathrm{GeV}$, which corresponds to beam energies beyond $2 \mathrm{GeV}$.

For beam energies beyond $1.5 \mathrm{GeV}(\sqrt{s}=2.5 \mathrm{GeV})$ we face substantial contributions from the $\rho^{0}$ decay of higherlying $N^{*}$ and $\Delta$ resonances, which get excited during the collision process. These sources contribute to the dilepton spectra both from $p n$ and $p p$ collisions as demonstrated by refs. $[5,7]$, who succeed in a quantitative description of the data for beam energies of $2 \mathrm{GeV}$ and beyond.

\section{Conclusions}

Since the $t$-channel $\Delta \Delta$ mechanism is the leading two-pion production process in $N N$ collisions at beam energies of $1-1.5 \mathrm{GeV}$, it is also the main source for $e^{+} e^{-}$production originating from $\rho^{0}$-channel $\pi^{+} \pi^{-}$production at these energies. This mechanism, however, contributes only in case of $n p$ collisions and vanishes in case of $p p$ collisions.

It has been shown that the $e^{+} e^{-}$production resulting from on-shell $\rho^{0}$-channel $\pi^{+} \pi^{-}$production gives contributions to the $p n$-initiated dilepton spectrum for $M_{e^{+} e^{-}}>$ $2 m_{\pi}$, which account very well for the missing strength in previous interpretations offering thus a possible solution of the long-standing DLS puzzle.

We are grateful to T. Galatyuk and M. Gumberidze for their help with HADES data and filtering software. We also want to thank P. Salabura for stimulating and fruitful discussions, in particular for drawing our attention to this issue. We are indebted to L. Alvarez-Ruso for using his code. We also acknowledge valuable discussions with A. Gal, T. Gutsche, C. Hanhart, J. Weil and C. Wilkin. This work has been supported by the Forschungszentrum Jülich (COSY-FFE).

Open Access This is an open access article distributed under the terms of the Creative Commons Attribution License (http://creativecommons.org/licenses/by/4.0), which permits unrestricted use, distribution, and reproduction in any medium, provided the original work is properly cited.

\section{References}

1. DLS Collaboration (R.J. Porter et al.), Phys. Rev. Lett. 79, 1229 (1997).

2. DLS Collaboration (W.K. Wilson et al.), Phys. Rev. C 57, 1865 (1998)

3. HADES Collaboration (G. Agakichiev et al.), Phys. Lett. B 690, 118 (2010).

4. R. Shyam, U. Mosel, Phys. Rev. C 82, 062201 (2010).

5. J. Weil, H. van Hees, U. Mosel, Eur. Phys. J. A 48, 111 (2012) 48, 150(E) (2012).

6. B.V. Martemyanov, M.I. Krivoruchenko, Amand Faessler, Phys. Rev. C 84, 047601 (2011).

7. E.L. Bratkovskaya, J. Aichelin, M. Thomere, S. Vogel, M. Bleicher, Phys. Rev. C 87, 064907 (2013).

8. L.P. Kaptari, B. Kämpfer, Nucl. Phys. A 764, 338 (2006).

9. PROMICE/WASA Collaboration (J. Johanson et al.), Nucl. Phys. A 712, 75 (2002). 
10. PROMICE/WASA Collaboration (W. Brodowski et al.), Phys. Rev. Lett. 88, 192301 (2002).

11. PROMICE/WASA Collaboration (J. Pätzold et al.), Phys. Rev. C 67, 052202 (2003).

12. CELSIUS/WASA Collaboration (T. Skorodko et al.), Eur. Phys. J. A 35, 2008 (317).

13. CELSIUS/WASA Collaboration (T. Skorodko et al.), Phys. Lett. B 679, 30 (2009).

14. CELSIUS/WASA Collaboration (F. Kren et al.), Phys. Lett. B 684, 110 (2010) 702, 312(E) (2011) arXiv:0910.0995v2 [nucl-ex].

15. CELSIUS/WASA Collaboration (T. Skorodko et al.), Phys. Lett. B 695, 115 (2011).

16. CELSIUS/WASA Collaboration (T. Skorodko et al.), Eur. Phys. J. A 47, 108 (2011).

17. WASA-at-COSY Collaboration (P. Adlarson et al.), Phys. Lett. B 706, 256 (2011).

18. COSY-TOF Collaboration (S. Abd El-Bary et al.), Eur. Phys. J. A 37, 267 (2008).

19. COSY-TOF Collaboration (S. Abd El-Samad et al.), Eur. Phys. J. A 42, 159 (2009).

20. CELSIUS/WASA Collaboration (M. Bashkanov et al.), Phys. Rev. Lett. 102, 052301 (2009).
21. WASA-at-COSY Collaboration (P. Adlarson et al.), Phys. Rev. Lett. 106, 242302 (2011).

22. WASA-at-COSY Collaboration (P. Adlarson et al.), Phys. Lett. B 721, 229 (2013).

23. WASA-at-COSY Collaboration (P. Adlarson et al.), Phys. Rev. C 88, 055208 (2013).

24. L. Alvarez-Ruso, E. Oset, E. Hernandez, Nucl. Phys. A 633, 519 (1998).

25. Xu Cao, Bing-Song Zou, Hu-Shan Xu, Phys. Rev. C 81, 065201 (2010).

26. WASA-at-COSY Collaboration (P. Adlarson et al.), Phys. Rev. Lett. 112, 202301 (2014).

27. L.G. Dakhno et al., Sov. J. Nucl. Phys. 37, 540 (1983).

28. J. Bystricky et al., J. Phys. 48, 1901 (1987).

29. F. Dohrmann et al., Eur. Phys. J. A 45, 401 (2010).

30. G. Fäldt, C. Wilkin, Phys. Lett. B 701, 619 (2011).

31. M. Albadejo, E. Oset, Phys. Rev. C 88, 014006 (2013).

32. A. Gal, H. Garcilazo, Phys. Rev. Lett. 111, 172301 (2013).

33. A. Gal, H. Garcilazo, doi:10.1016/j.nuclphysa.2014.02.019.

34. C.Q. Li, C.M. Ko, G.E. Brown, Phys. Rev. Lett. 75, 4007 (1995).

35. P. Koch, Z. Phys. C 57, 283 (1993).

36. Particle Data Group (J. Beringer et al.), Phys. Rev. D 86, 010001 (2012). 